Historic, Archive Document

Do not assume content reflects current scientific knowledge, policies, or practices. 


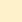




\section{IRIS GERMANICA \\ THE FLEUR DE LIS OF FRANCE PRICE LIST}

FALL 1921 AND SPRING 1922

2

The Prices for single plants quoted in this list are the same as previous seasons, as one cannot supply real first-class plants at a less

price if you want to get paid for your time and trouble

in making out small orders. Prices by the ten

and 100 are considerable reduced

from prices quoted 1920.

You will not find the latest novelties in this list. Past experience has taught me that a good many of the new irises are tender, and cost more as they are worth. 
Prices quoted are for extra strong plants; 5 per rate of 10 ; single plants postage prepaid. Terms cash.

ADMIRAL TOGO: S. \& F. white bordered lavender.

Each Ten 100 same habit as Mad Chereau, extra fine 36 -inch...\$\$.20 $\$ \$ 1.00 \quad \$ 4.50$

ALBER'T VICTOR: S. \& F. soft lavender blue, vigorous grower, one of the best of the Pallida section.

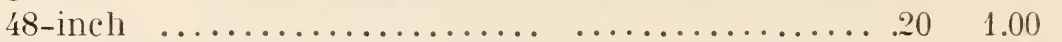

BLACK PRINCE: S. Purple Lilac; F. velvety black, an

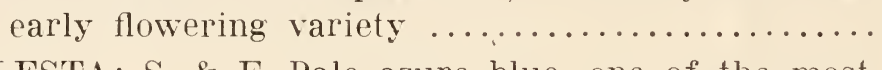

CELESTA: S. \& F. Pale azure blue, one of the most beautiful

CYPRIANA: S. Pale blue, F. Iong, rich purple re-

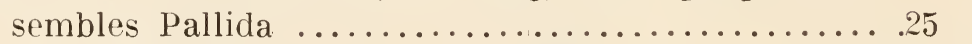

DR. BERNICE: S. Coppery bronze; F. velvety crimson, large and beautiful. Extra fine 24-inch........ 30 $1.50 \quad .500$ GERTRUDE: S. \& F. Violet blue, an early free

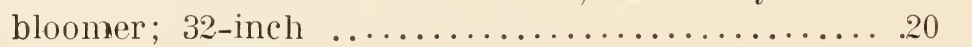

HARLEQUIN MELANOIS: S. \& F. white and blue; 32-inch; one of the most curious mottled of all

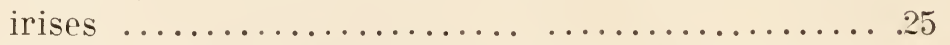

INGEBORG: S. \& F. pure white, large flowers, the best white in the trade; fine for cut flowers; 24-inch... . 25

INNOCENZA: S. \& F. ivory white, crest rich golden;

a fine white variety.....................25 1.50

IRIS KING: S. lemon yellow; F. maroon bordered yellow; a very beautiful new variety; 34 -inch.....

ISOLINE: S. lilac pink; F. purplish rose; one of the grandest varieties in cultivation, 36 -inch......... JACQUESIANA: S. bright copper crimson; F. bright maroon: distinct one of the best reds, 36 -inch..... .50 3.50 15.00 .50 3.00 12.00

JOHN De WERTH: S. light mauve; F. purple, heavily

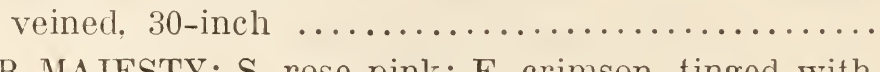
$.30 \quad 2.00$

HER MAJESTY: S. rose pink; F. crimson, tinged with darker shade, $32-i n c h \ldots \ldots \ldots \ldots \ldots \ldots \ldots \ldots \ldots . .30$

LEONIDAS: S. violet blue; F. rich velvety purple, late

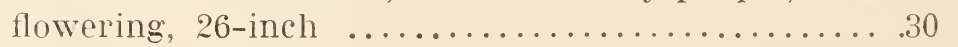
2.00

MACRANTHA: (Syn. Amas) S. rich blue; F. violet, early flowering, extra fine and large, 24-inch..... 
MAD CHEREAU: S. \& F. white frilled with a wide border of clear blue, extra fine, 34 -inch........ $\$ .20 \quad \$ 1.50$

MAD PACQUETTE: S. \& F. bright rosy red, distinct flowers produced freely ................... .20 1.50

OTHELLO: S. deep rich blue; F. dark velvety purple,

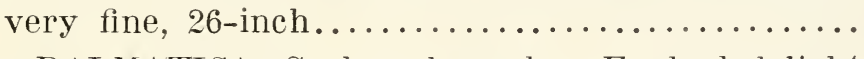

PAL. DALMATICA: S. deep lavender; F. shaded light purple, a grand variety, large flowers...........

PAL. MANDRALISCOE: F. \& S. rich lavender purple, tall, large and handsome, 40-inch............30

PAL. SPECIOSA: S. dark lavender shaded lighter;

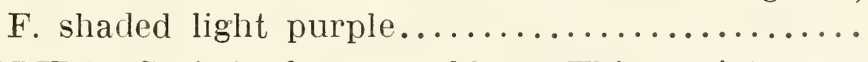
$.30 \quad 2.00$

PALLIDA: S. \& F. lavender blue. This variety was bought over 50 years ago as Agathocles. The The strongest grower of this section, leaves 2

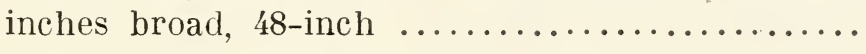

PARISENSIS: S. purple lilac; F. rich velvety blue,

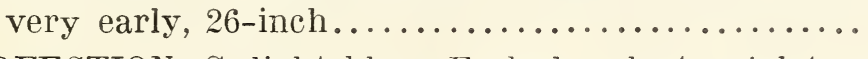

PERFECTION: S. light blue; F. dark velvety violet, a handsome abundant bloomer, 32-inch......... .25

PRINCESS VICTORIA: S. sulphur yellow; F. plum color, bordered cream, extra fine, 32-inch........

QUEEN of MAY: S. \& F. soft rosy lilac shaded pink,

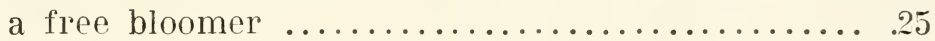

REEINNIXE: S. pure white; F. violet blue with an edge, very handsome, extra fine, 32 -inch......... .35

2.50

TAMERLANE: S. pale purple; F. deep violet purple, very large, a handsome variety, 36 -inch.......... $.35 \quad 2.50$

The following new varieties I can supply only in single plants as they did not increase any on account of the drought.

ARCHEVEQUE: S. rich purple; F. deep velvety violet; extreme rich coloring, 24-inch.............. .50

CAPRICE: reddish purple; F. deeper purple, large and beautiful, 36 -inch .....................50

CATERINA: Claimed to be an improved Dalmatica; a high-priced iris, likes to rot..................

ELDORADO: S. bronze shaded yellow; F. purple bordered yellow ......................50 
LOHENGRIN: S. \& F. light rose, large and handsome, vigorous grower $\ldots \ldots \ldots \ldots \ldots \ldots \ldots \ldots \ldots \ldots$ MONSIGNOR: S. lavender; F. velvety purple crimson,

a large handsome variety, 24 -inch.............75 PRINCESS TICTORIA: S. \& F. pure white, 32-inch... .50 QUEEN EMMA: S. \& F. clear waxy white.......... . 50 TWILIGHT: S. \& F. delicate violet purple........ . 50 VICTORINE: S. white; F. mottled violet........... .30 WYOMISSING: S. rosy white; F. deep rose shading

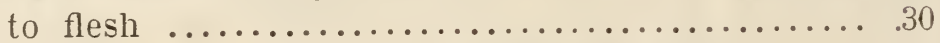

\section{SIBERIAN IRIS}

The Siberian Irisis have narrow grassy foliage with beautiful flowers. Fine for cutting. SIBERIAN BLUE ..................... \$.25

SIBERIAN GRANDIS: Violet reticulated white..... .25 SIBERIAN ORIENTALIS: Deep blue. large for its class .25 SIBERIAN SUPERBA: Violet blue, handsome foliage. . 25 SIBERIAN SNOW QUEEN: Ivory white flowers..... .25

All orders are accepted on condition. that I am not sold out of varieties wanted, and they shall be void, should injury befall my stock from frost, fire, or other cause, over which I have no control. All varieties are guaranteed true to name. Mr Gladioli and Dahlia catalogue will be issued next December. Watch out for something new in that line.

\section{THE FLOWER GROWER}

(Formerly the Modern Gladiolus Grower)

Published Monthly by Madison Cooper at Calcium, N. Y. Subscription Price: Three Years, $\$ 2.00$; One Year, $\$ 1.00$. 